\title{
Multi-scenario based robust intensity-modulated proton therapy (IMPT) plans can account for set-up errors more effectively in terms of normal tissue sparing than planning target volume (PTV) based intensity-modulated photon plans in the head and neck region
}

Martin Stuschke ${ }^{1,2^{*}}$, Andreas Kaiser ${ }^{1,2}$, Jehad Abu Jawad ${ }^{1}$, Christoph Pöttgen ${ }^{1}$, Sabine Levegrün ${ }^{1+}$ and Jonathan Farr ${ }^{2,3+}$

\begin{abstract}
Background: In a previous report, we compared the conformity of robust intensity-modulated proton therapy (IMPT) plans with that of helical tomotherapy plans for re-irradiations of head and neck carcinomas using a fixed set-up error of $2 \mathrm{~mm}$. Here, we varied the maximum set-up errors between 0 and $5 \mathrm{~mm}$ and compared the robust IMPT-plans with planning target volume (PTV) based intensity-modulated photon therapy (IMRT).

Findings: Seven patients were treated with a PTV-based tomotherapy plan. Set-up margins of 0, 2, and $5 \mathrm{~mm}$ were subtracted from the PTV to generate target volumes (TV) $T V_{0 m m}, T V_{2 m m}$, and $T V_{5 m m}$, for which robust IMPT-plans were created assuming range uncertainties of $\pm 3.5 \%$ and using worst case optimization assuming set-up errors of 0 , 2 , and $5 \mathrm{~mm}$, respectively. Robust optimization makes use of the feature that set-up errors in beam direction alone do not affect the distal and proximal margin for that beam. With increasing set-up errors, the body volumes that were exposed to a selected minimum dose level between $20 \%$ and $95 \%$ of the prescribed dose decreased. In IMPTplans with $0 \mathrm{~mm}$ set-up error, the exposed body volumes were on average $6.2 \% \pm 0.9 \%$ larger than for IMPT-plans with $2 \mathrm{~mm}$ set-up error, independent of the considered dose level $(p<0.0001$, F-test). In IMPT-plans accounting for $5 \mathrm{~mm}$ set-up error, the exposed body volumes were by $11.9 \% \pm 0.8 \%$ smaller than for IMPT-plans with $2 \mathrm{~mm}$ set-up error at a fixed minimum dose ( $p<0.0001$, F-test). This set-up error dependence of the normal tissue exposure around the TV in robust IMPT-plans corresponding to the same IMRT-plan led to a decrease in the mean dose to the temporal lobes and the cerebellum, and in the D2\% of the brain stem or spinal cord with increasing set-up errors considered during robust IMPT-planning.
\end{abstract}

Conclusions: For recurrent head and neck cancer, robust IMPT-plan optimization led to a decrease in normal tissue exposure with increasing set-up error for target volumes corresponding to the same PTV.

Keywords: Intensity modulated proton therapy, IMPT, Robust optimization, Head and neck cancer, Re-irradiation

\footnotetext{
* Correspondence: martin.stuschke@uk-essen.de

${ }^{\dagger}$ Equal contributors

'Department of Radiotherapy, University Hospital Essen, Essen 45147, Germany

West German Proton, Therapy Centre Essen, Essen 45147, Germany

Full list of author information is available at the end of the article
} 


\section{Background}

The consideration of a $2 \mathrm{~mm}$ set-up error seems adequate for small targets in the head and neck region when daily online navigation is used [1,2]. However, in the head and neck region, less frequent online correction protocols and off-line protocols correcting only systematic set-up errors may require margins between the clinical target volume (CTV) and the planning target volume (PTV) accounting for larger set-up errors of $5 \mathrm{~mm}$ or more $[3,4]$.

Robust intensity-modulated proton therapy (IMPT) treatment plan optimization, optimizing the dose distribution in the worst case of multiple scenarios covering possible realizations of set-up errors and density variations, can result in treatment plans that maintain target volume coverage and normal tissue sparing in the presence of set-up errors and range uncertainties [5-10]. A single scenario PTV based treatment planning may not be sufficient for IMPT because possible spatial deviations of dose gradients between spots can affect dose homogeneity within the PTV with set-up error $[5,11]$.

In a previous study, we compared dose conformity and normal tissue exposure of intensity-modulated photon plans for helical tomotherapy with robust IMPT-plans for set-up errors of $2 \mathrm{~mm}$ [6]. Here, we test the performance of the robust multi-scenario based IMPT optimization to spare normal tissues around a given PTV per patient for different set-up errors varying between 0 and $5 \mathrm{~mm}$. With increasing set-up errors, the CTV-PTV set-up margin contains an increasing portion of the PTV and robust IMPT optimization based on the CTV may lead to results which depend systematically on the set-up margin size.

\section{Findings \\ Methods \\ Dataset}

The analysis was based on 7 patients with recurrent head and neck carcinomas who were re-irradiated using helical tomotherapy. Patients and tomotherapy treatment planning were described in a previous publication [6].

\section{Intensity-modulated proton therapy planning}

Target volumes $T V_{0 \mathrm{~mm}}, \mathrm{TV}_{2 \mathrm{~mm}}$, and $T V_{5 \mathrm{~mm}}$ were obtained from the original tomotherapy PTV of each patient by concentric shrinkage by 0,2 , and $5 \mathrm{~mm}$, respectively. IMPT-plans were generated to cover $\mathrm{TV}_{0 \mathrm{~mm}}$, $\mathrm{TV}_{2 \mathrm{~mm}}$, and $\mathrm{TV}_{5 \mathrm{~mm}}$ using the field configurations described in the previous study [6]. Robust optimization was used assuming a range, i.e. density, uncertainty of $+/-3.5 \%$. In addition, maximum set-up errors of 0,2 , and $5 \mathrm{~mm}$ in each direction were considered in the robust optimization of the IMPT-plans based on $\mathrm{TV}_{0 \mathrm{~mm}}$,
$\mathrm{TV}_{2 \mathrm{~mm}}$, and $\mathrm{TV}_{5 \mathrm{~mm}}$, respectively. IMPT planning was performed using the RayStation v2.4.13.31 planning system as described by Fredrikson et al. [7]. Robust optimization evaluates the penalties of the original plan and the plans with shifted beam isocenters corresponding to the maximum set-up errors and altered density scalings. Optimization of the pencil beam spot weights is performed to minimize the penalties of the worst case scenario. In a previous publication, IMPT-plans assuming set-up errors of $2 \mathrm{~mm}$ and using $\mathrm{TV}_{2 \mathrm{~mm}}$ as CTV were compared to tomotherapy plans optimized on the PTV, i.e. $\mathrm{TV}_{0 \mathrm{~mm}}[6]$. In the present study, we compared, for every patient, the robust IMPT-plans based on $\mathrm{TV}_{0 \mathrm{~mm}}, \mathrm{TV}_{2 \mathrm{~mm}}$, and $\mathrm{TV}_{5 \mathrm{~mm}}$, assuming maximum set-up errors of 0,2 , and $5 \mathrm{~mm}$, respectively $\left(\mathrm{IMPT}_{0 \mathrm{~mm}}\right.$, $\mathrm{IMPT}_{2 \mathrm{~mm}}$, and $\mathrm{IMPT}_{5 \mathrm{~mm}}$, respectively) with the original PTV-based tomotherapy photon plan. Differences in the dose distributions of the $\mathrm{IMPT}_{\mathrm{Omm}^{-}}, \mathrm{IMPT}_{2 \mathrm{~mm}^{-}}$, and $\mathrm{IMPT}_{5 \mathrm{~mm}}$-plans reflect the performance of a robust scenario-based optimization to handle set-up errors in comparison to a PTV-based approach.

\section{Statistical analysis}

The effect of the set-up error on the body volume exposed to doses higher than $\mathrm{x} \%$ of the prescribed dose ( $\mathrm{V}_{\text {body }} \mathrm{x} \%$ ), where $20 \% \leq \mathrm{x} \% \leq 95 \%$, in the IMPT-plans $\mathrm{IMPT}_{0 \mathrm{~mm}}, \mathrm{IMPT}_{2 \mathrm{~mm}}$, and $\mathrm{IMPT}_{5 \mathrm{~mm}}$, respectively, was analyzed using Proc Glm, SAS statistical software Version 9.2 (Cary, NC). The logarithm of the ratio of $\mathrm{V}_{\text {body }} \mathrm{x}$ $\%$ exposed in plan $\mathrm{IMPT}_{\mathrm{ymm}}$ to $\mathrm{V}_{\text {body }} \mathrm{x} \%$ exposed in plan $\mathrm{IMPT}_{2 \mathrm{~mm}}$ (i.e. $\log \left(\mathrm{V}_{\text {body }} \mathrm{x} \%\right.$ in $\mathrm{IMPT}_{\text {ymm }} / \mathrm{V}_{\text {body }} \mathrm{x} \%$ in $\mathrm{IMPT}_{2 \mathrm{~mm}}$ ) was used as dependent variable, where ymm denotes a set-up error of y $\mathrm{mm}$. The set-up error was used as an independent classification variable, the individual patient as a random variable, and the dose level $\mathrm{x}$ and the square of the dose level $\mathrm{x} \cdot \mathrm{x}$ as continuous regressor variables. Dose differences to organs at risk in the $\mathrm{IMPT}_{\mathrm{ymm}}$-plans compared with the $\mathrm{IMPT}_{2 \mathrm{~mm}}$-plans were analyzed in a similar manner.

\section{Results}

\section{Exposed body volumes}

For all evaluated isodose levels $\mathrm{x}$ between 20\% and 95\%, $\mathrm{V}_{\text {body }} \mathrm{x} \%$ exposed in the robust $5 \mathrm{~mm}$ plans $\left(\mathrm{IMPT}_{5 \mathrm{~mm}}\right.$ ) was on average smaller (mean: $-11.9 \%, 95 \%$ confidence interval of the mean: $-13.4 \%$ to $-10.3 \%, \mathrm{p}<0.0001$, F-test) than $\mathrm{V}_{\text {body }} \mathrm{x}$ exposed in the robust $2 \mathrm{~mm}$ plans $\left(\mathrm{IMPT}_{2 \mathrm{~mm}}\right)$. Compared with the $\mathrm{IMPT}_{2 \mathrm{~mm}}$-plans, a $6.2 \%$ increase of the exposed body volume $\mathrm{V}_{\text {body }} \mathrm{x} \%$ was obtained in the $0 \mathrm{~mm}$ plans $\left(\mathrm{IMPT}_{0 \mathrm{~mm}}\right)$, averaged over all patients and isodose levels (95\% confidence interval: 4.4\% to $8.1 \%, \mathrm{p}<0.0001$, F-test). The deviations of the body volumes exposed in the $\mathrm{IMPT}_{5 \mathrm{~mm}}$ - and $\mathrm{IMPT}_{0 \mathrm{~mm}}$-plans from the body volume exposed in the $\mathrm{IMPT}_{2 \mathrm{~mm}}$-plan did not 
depend on the selected isodose level $\mathrm{x}$, because neither a linear nor a quadratic term of $\mathrm{x}$ became significant using a Taylor series expansion ( $p>0.25$ for each term). Figure 1 shows the ratios of $\mathrm{V}_{\text {body }} \mathrm{x}$ exposed in the $\mathrm{IMPT}_{5 \mathrm{~mm}^{-}}$ and $\mathrm{IMPT}_{0 \mathrm{~mm}}$-plans over $\mathrm{V}_{\text {body }} \mathrm{x} \%$ exposed in the $\mathrm{IMPT}_{2 \mathrm{~mm}}$-plan for all 7 patients and all evaluated isodose levels $\mathrm{x}$ between $20 \%$ and $95 \%$. There was a significant inter-patient variability in the mean increase of the exposed body volume for the $\mathrm{IMPT}_{0 \mathrm{~mm}}$-plans compared with the $\mathrm{IMPT}_{2 \mathrm{~mm}}$-plans over $\mathrm{V}_{\text {body }} \mathrm{x} \%(\mathrm{p}<0.0001, \mathrm{~F}-\mathrm{Test})$. The smallest increase amounted to $1.2 \%$ (patient 6), the largest increase was $19.0 \%$ (patient 1) (mean increase: $+6.2 \%$ ). The inter-patient variability in the decrease of the exposed body volume for the $\mathrm{IMPT}_{5 \mathrm{~mm}}$-plans compared with the $\mathrm{IMPT}_{2 \mathrm{~mm}}$-plans was also significant $(\mathrm{p}<0.0001, \mathrm{~F}$-Test). The smallest decrease was $-6.9 \%$ (patient 6 ), the largest decrease amounted to $-18.6 \%$ (patient 1) (mean decrease: $-11.9 \%$ ). Average depths of the proximal and distal CTV border at the isocenter were $3.7 \mathrm{~cm}$ and $8.6 \mathrm{~cm}$, respectively, over all coplanar fields for the considered patients with larger inter-field variations. The corresponding range uncertainties were $1.3 \mathrm{~mm}$ and $3.0 \mathrm{~mm}$ in this study.

Figure 2 shows dose difference plots calculated by subtracting the dose distribution of the $\mathrm{IMPT}_{2 \mathrm{~mm}}$-plan from the dose distribution of the $\mathrm{IMPT}_{0 \mathrm{~mm}^{-}}$and

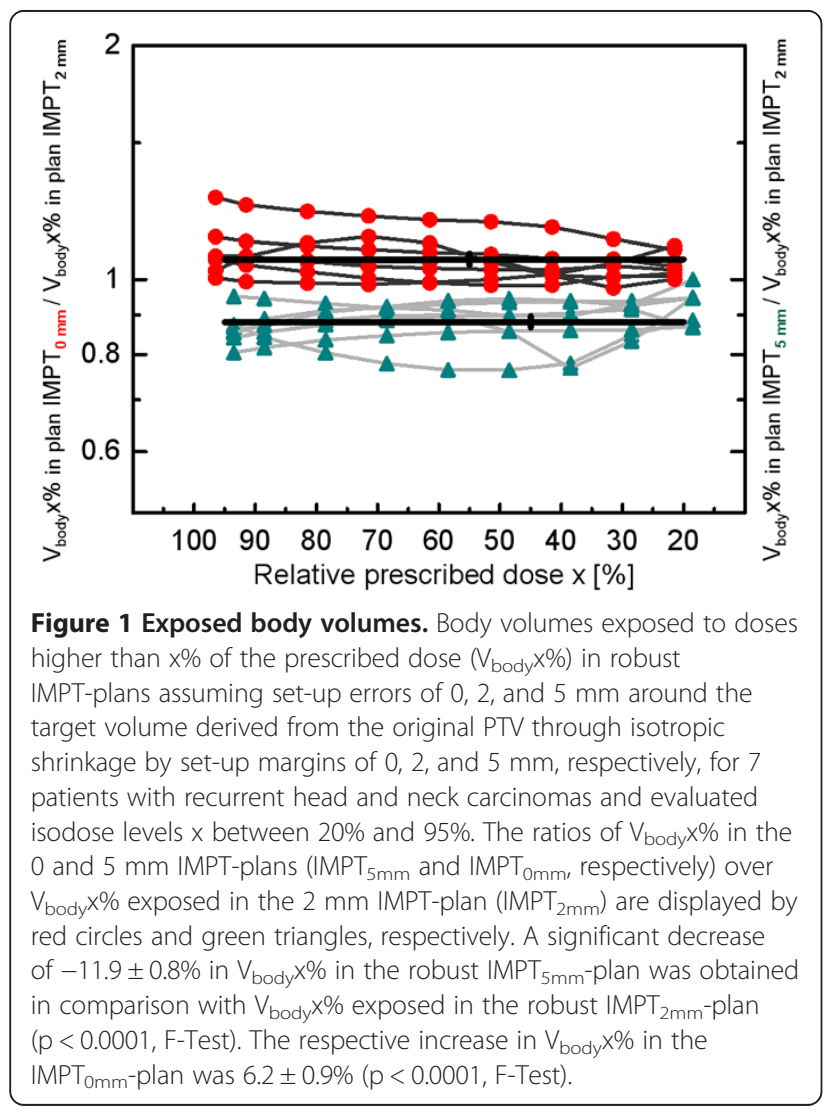

$\mathrm{IMPT}_{5 \mathrm{~mm}}$-plans for patients 1 and 7, respectively. These dose difference distributions demonstrate the increase of the dose around the target volume in the $0 \mathrm{~mm}$ IMPTplan and the decrease of the dose in the $5 \mathrm{~mm}$ IMPTplan compared with the $2 \mathrm{~mm}$ IMPT-plan. On average, the $\mathrm{TV}_{0 \mathrm{~mm}}$-size was $20 \%$ larger and the $\mathrm{TV}_{5 \mathrm{~mm}}$-size $37 \%$ smaller than the size of the $\mathrm{TV}_{2 \mathrm{~mm}}$. The absolute values are given in [6].

The coverage of the robust IMPT-plans for 0,2 , and $5 \mathrm{~mm}$ set-up errors remained high with respect to the relevant target volumes $\mathrm{TV}_{0 \mathrm{~mm}}, \mathrm{TV}_{2 \mathrm{~mm}}$, and $\mathrm{TV}_{5 \mathrm{~mm}}$. In the $\mathrm{IMPT}_{0 \mathrm{~mm}}$-plans, the dose to $95 \%$ of the target volume was $>98 \%$ of the prescribed dose for patients 1 and 6 and $>100 \%$ of the prescribed dose for all other patients. In the $\mathrm{IMPT}_{2 \mathrm{~mm}^{-}}$and $\mathrm{IMPT}_{5 \mathrm{~mm}}$-plans, the dose to $95 \%$ of the target volume was $>95 \%$ of the prescribed dose for patient 1 and $>100 \%$ for all other patients. The robustness against diagonal set-up errors was tested for the 2 and $5 \mathrm{~mm}$ IMPT-plans by shifting the plan isocenter by 1.5 or $3.5 \mathrm{~mm}$ in the lateral and ventrodorsal directions and applying a density change of $+3 \%$. The $\mathrm{IMPT}_{5 \mathrm{~mm}}$-plans were as robust against combined set-up errors of $3.5 \mathrm{~mm}$ in the directions specified above as the $\mathrm{IMPT}_{2 \mathrm{~mm}}$-plan against set-up errors of $1.5 \mathrm{~mm}$.

\section{Normal tissue exposure}

Dose difference distributions between the $\mathrm{IMPT}_{0 \mathrm{~mm}^{-}}$ and the IMPT $\mathrm{Imm}_{2 \mathrm{~m}}$-plans and between the $\mathrm{IMPT}_{5 \mathrm{~mm}}$ and the $\mathrm{IMPT}_{2 \mathrm{~mm}}$-plans were also analyzed with respect to the exposure of selected normal tissues and organs at risk. Differences in the mean doses to the ipsilateral temporal lobe and to the cerebellum in the $\mathrm{IMPT}_{0 \mathrm{~mm}^{-}}$ and $\mathrm{IMPT}_{5 \mathrm{~mm}}$-plans compared with the $\mathrm{IMPT}_{2 \mathrm{~mm}}$-plan are shown in Figure 3 for the 7 patients. The mean doses to the ipsilateral temporal lobe and to the cerebellum showed a dependence on the size of the set-up error considered in the robust optimization $(\mathrm{p}=0.009$ and $\mathrm{p}=$ 0.001 , F-tests). Smaller mean doses were obtained for larger set-up errors considered. The same holds for the D2\%-differences in the brain stem and spinal cord between the $\mathrm{IMPT}_{0 \mathrm{~mm}}$ - and the IMPT $\mathrm{Imm}_{5 \mathrm{~m}}$-plans compared with the $\mathrm{IMPT}_{2 \mathrm{~mm}}$-plan (Figure 3). Considering the serial organ with the highest D2\% in each patient, either the brainstem or the spinal cord, the D2\%-difference to the $\mathrm{IMPT}_{2 \mathrm{~mm}}$-plan was significantly larger for the $\mathrm{IMPT}_{0 \mathrm{~mm}}$-plan compared with the $\mathrm{IMPT}_{5 \mathrm{~mm}}$-plan $(\mathrm{p}=$ 0.016, F-test).

\section{Discussion}

Set-up margins alone cannot ensure dose coverage of the CTV in IMPT-plans with multiple fields and high in-field dose gradients [5,11]. Deviations in the position of these dose gradients in the patient from spot to spot due to set-up errors or range uncertainties can result in 


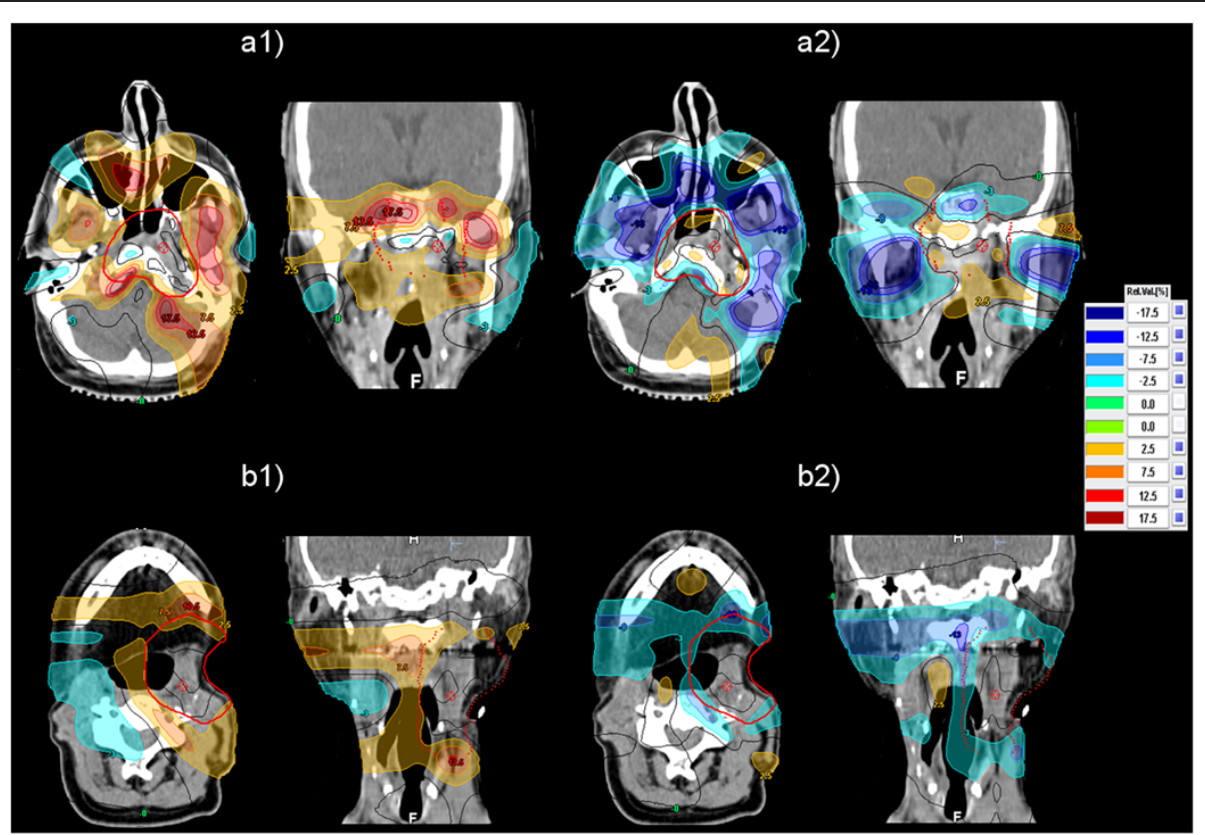

Figure 2 Dose difference plots. Distribution of the dose difference obtained by subtracting the dose distribution of the IMPT $2 \mathrm{~mm}-\mathrm{plan}$ from the dose distributions of the IMPT $0 \mathrm{~mm}^{-}$and the IMPT $5 \mathrm{~mm}$-plans. (a1) Dose difference IMPT $0 \mathrm{~mm}-\mathrm{IMPT}_{2 \mathrm{~mm}}$ for patient 1. (a2) Dose difference IMPT $5 \mathrm{~mm}^{-}$ $\mathrm{IMPT}_{2 \mathrm{~mm}}$ for patient 1. The corresponding dose difference plots for patient 7 are shown in (b1) and (b2), respectively.

under- or overdosage inside the PTV. Robust IMPT optimization can lead to smooth dose gradients per field across a target volume and can diminish the risk of pencil beams stopping directly in front of an abutting normal tissue $[5,8,9]$. It makes use of the feature that set-up errors in beam direction alone do not affect the distal and proximal margin for that beam as also a beamspecific PTV-margin concept would do [12] but unlike

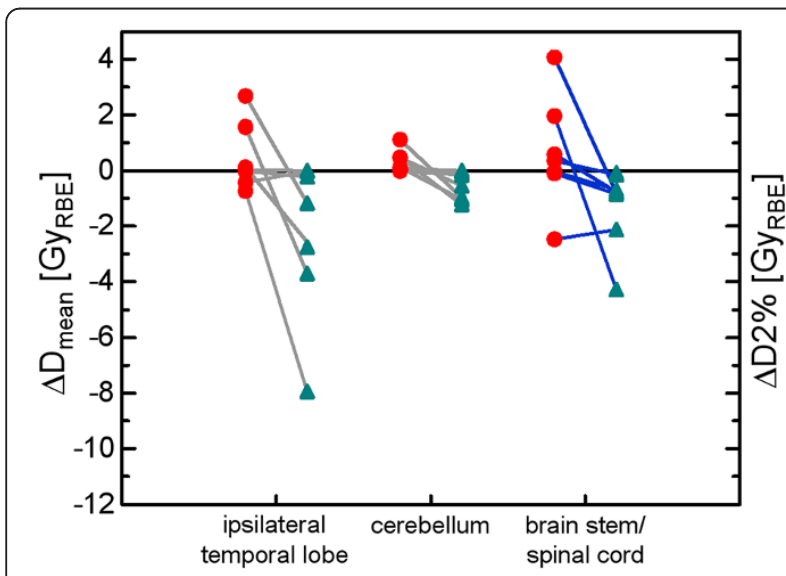

Figure 3 Normal tissue exposure. Mean dose difference to the ipsilateral temporal lobe and the cerebellum between the IMPT $\mathrm{Mmm}_{-}$ and IMPT $2 \mathrm{~mm}$-plans (red circles) and between the IMPT $\mathrm{mm}^{-}$and $\mathrm{IMPT}_{2 \mathrm{~mm}}$-plans (green triangles) for the 7 patients (left vertical axis). The respective differences in D2\%, quantifying hot spots in the serial organ of each patient exposed to the highest doses, i.e. the either brain stem or the spinal cord, are also shown (right vertical axis). the PTV concept of expanding the CTV by set-up margin used in photon therapy. Scanned particle beams can spare normal tissues in beam direction behind the target volume and conform to the proximal contour of the target volume so that a reduction of normal tissue exposure per beam can result. Unlike CTV expansion by set-up margins, the robust IMPT optimization method also considers the potential influence of lateral set-up errors on the water equivalent depth of the distal and proximal CTV border at a given lateral spot position due to lateral density inhomogeneities in the entrance channel, e.g. if bone is moved in front of the target volume at that spot position due to set-up error. Multi-scenario based robust IMPT-plans can maintain dose coverage of the target volume better than PTV-based IMPT-plans $[5,13]$. On the other hand, the spatial dose distribution can be considered as independent of small set-up errors and organ motions for rotational or fixed-field intensity-modulated photon therapy [14]. Thus, the PTV concept is usually adopted to account for set-up errors in intensitymodulated photon therapy. In this study, we showed that for recurrent head and neck carcinomas robust IMPT-plans perform the better relative to a photon intensity-modulated plan the larger the set-up margin as a part of the PTV is. This holds true for highly constrained IMPT field arrangements with up to 7 fields. Therefore, with increasing set-up margin portion of the PTV due to larger set-up errors, robust optimization can increase the on average lower conformity of IMPT in 
comparison with helical tomotherapy at or above the $50 \%$ isodose found in the previous study [6] for $2 \mathrm{~mm}$ set-up errors. Multiple scenarios, considered in robust optimization could also by linked by elastic deformations in the future to simulate internal motion and body deformation. The magnitude of range uncertainties assumed might also vary slightly from institution to institution [15].

\section{Conclusion}

Unlike the PTV-approach for photon IMRT, robust multi-scenario based IMPT optimization can increasingly reduce the normal tissue exposure around the target volume for recurrent head and neck cancer with increasing set-up margin portion of the PTV.

\section{Competing interests}

Parts of this work were supported by Grant No. STU-151/9-1 from the German Research Foundation (Deutsche Forschungsgemeinschaft). The authors declare that they have no competing interests.

\section{Authors' contributions}

MS carried out the plan comparisons, performed the statistical analysis and drafted the manuscript. He also contributed to the optimization of both helical tomotherapy and proton therapy plans. AK performed the robust optimization and evaluation of the IMPT-plans. He reviewed and edited draft versions of the paper. JAJ and CP conducted clinical treatment of the patients, generated initial volumes of interest, and approved the clinical helical tomotherapy plans. SL performed the helical tomotherapy optimization and the dosimetric analysis of both tomotherapy and IMPTplans. She also reviewed and edited draft versions of the paper. JF was involved in improving the proton therapy optimization approaches and in the design of the proton therapy planning system. He also participated in concept discussions for developing the investigative aims and critically reviewed the draft manuscript. All authors read and approved the final manuscript.

\section{Author details}

'Department of Radiotherapy, University Hospital Essen, Essen 45147 Germany. ${ }^{2}$ West German Proton, Therapy Centre Essen, Essen 45147, Germany. ${ }^{3}$ Current address: Department of Radiologic Sciences, St. Jude Children's Research Hospital, Memphis, TN, USA.

Received: 3 June 2013 Accepted: 13 June 2013

Published: 18 June 2013

\section{References}

1. Levegrün S, Pöttgen C, Abu Jawad J, Berkovic K, Hepp R, Stuschke M: Megavoltage computed tomography Image guidance with helical tomotherapy in patients with vertebral tumors: analysis of factors influencing interobserver variability. Int I Radiat Oncol Biol Phys 2013, 85:561-569

2. Schulte RW, Fargo RA, Meinass HJ, Slater JD, Slater JM: Analysis of head motion prior to and during proton beam therapy. Int I Radiat Oncol Biol Phys 2000, 47:1105-1110.

3. Houghton F, Benson RJ, Tudor GS, Fairfoul J, Gemmill J, Dean JC, Routsis DS, Jefferies SJ, Burnet NG: An assessment of action levels in imaging strategies in head and neck cancer using tomotherapy. are our margins adequate in the absence of image guidance? Clin Oncol (R Coll Radiol) 2009, 21:720-727.

4. Rudat V, Hammoud M, Pillay Y, Alardi AA, Mohamed A, Altuwaijri S: Impact of the frequency of online verifications on the patient set-up accuracy and set-up margins. Radiat Oncol 2011, 6:101. doi:10.1186/1748-717X-6-101.

5. Stuschke M, Kaiser A, Pöttgen C, Lübcke W, Farr J: Potentials of robust intensity modulated scanning proton plans for locally advanced lung cancer in comparison to intensity modulated photon plans. Radiother Oncol 2012, 104:45-51.
6. Stuschke M, Kaiser A, Abu Jawad J, Pöttgen C, Levegrün S, Farr J: Re-irradiation of recurrent head and neck carcinomas: comparison of robust intensity modulated proton therapy treatment plans with helical tomotherapy. Radiat Oncol 2013, 8:93.

7. Fredriksson A, Forsgren A, Hardemark B: Minimax optimization for handling range and setup uncertainties in proton therapy. Med Phys 2011, 38:1672-1684.

8. Pflugfelder D, Wilkens JJ, Oelfke D: Worst case optimization: a method to account for uncertainties in the optimization of intensity modulated proton therapy. Phys Med Biol 2008, 53:1689-1700.

9. Unkelbach J, Chan TCY, Brotfeld T: Accounting for range uncertainties in the optimization of intensity modulated proton therapy. Phys Med Biol 2007, 52:2755-2773.

10. Liu W, Zhang X, Li Y, Mohan R: Robust optimization of intensity modulated proton therapy. Med Phys 2012, 39:1079-1091.

11. Albertini F, Hug EB, Lomax AJ: Is it necessary to plan with safety margins for actively scanned proton therapy? Phys Med Biol 2011, 56:4399-4413.

12. Park PC, Zhi XR, Lee AK, Shoo N, Melancon AD, Zhang L, Dong L: A beamspecific planning target volume [PTV] design for proton therapy to account for setup and range uncertainties. Int I Radiat Oncol Biol Phys 2012, 82:e329-e336.

13. Liu W, Frank SJ, Li X, Li Y, Zhu RX, Mohan R: PTV-based IMPT optimization incorporating planning risk volumes vs robust optimization. Med Phys 2013, 40(2):021709. doi:10.1118/1.4774363.

14. Bortfeld T, Jokivarsi K, Goitein M, Kung J, Jiang SB: Effects of intra-fraction motion on IMRT dose delivery: statistical analysis and simulation. Phys Med Biol 2002, 47:2203-2220.

15. Paganetti $\mathrm{H}$ : Range uncertainties in proton therapy and the role of Monte Carlo simulations. Phys Med Biol 2002, 57:R99-R117.

doi:10.1186/1748-717X-8-145

Cite this article as: Stuschke et al:: Multi-scenario based robust intensitymodulated proton therapy (IMPT) plans can account for set-up errors more effectively in terms of normal tissue sparing than planning target volume (PTV) based intensity-modulated photon plans in the head and neck region. Radiation Oncology 2013 8:145.

\section{Submit your next manuscript to BioMed Central and take full advantage of:}

- Convenient online submission

- Thorough peer review

- No space constraints or color figure charges

- Immediate publication on acceptance

- Inclusion in PubMed, CAS, Scopus and Google Scholar

- Research which is freely available for redistribution 